\title{
BENEFICIOS DEL ENTRENAMIENTO MUSICAL PARA EL APRENDIZAJE DE UNA LENGUA EXTRANJERA
}

\section{María del Carmen Fonseca Mora}

Universidad de Huelva

fonseca@uhu.es

\section{Javier Ávila López}

Universidad de Córdoba javila@uco.es

\section{Arturo Gallego Segador}

Universidad de Córdoba

ma1gasea@uco.es

El creciente auge de publicaciones internacionales sobre la relación existente entre aptitud musical y aptitud lingüística sugiere que el entrenamiento musical pudiera favorecer el aprendizaje de lenguas extranjeras. El objetivo de este estudio fue analizar la relación entre formación musical y aprendizaje de inglés como lengua extranjera de 454 alumnos españoles de la ESO. En primera instancia, un estudio correlacional contrastó los resultados académicos de los participantes en un programa piloto que combina estudios musicales y enseñanza secundaria con otro grupo de estudiantes del mismo centro sin participación en el estudio piloto. En una segunda fase, se analizó el sentido de autoeficacia del alumnado y su intención de seguir estudiando el idioma. Los resultados obtenidos indican la existencia de una influencia estadísticamente significativa del entrenamiento musical instrumental en el aprendizaje de inglés como lengua extranjera.

Palabras clave: formación musical; aprendizaje de lenguas extranjeras; aptitud musical; aptitud lingüística

Current rise of international papers on the close link of musical aptitude and linguistic aptitude indicates that musical training may foster foreign language learning. The focus of this study was analyzing instrumental musical training and language learning in 454 Spanish learners of compulsory secondary education. A correlational study compared the academic success of students enrolled in a pilot musical programme combining secondary education and musical studies to that of students in the same school following the standard secondary education syllabus. In a second stage, the two variables "self-efficacy and willingness to keep studying English" were also considered. The analysis shows a statistically significant incidence of instrumental musical training on language learning as explained by higher level of self-efficacy and willingness to keep studying English.

Keywords: musical training; foreign language learning; musical aptitude; linguistic aptitude

\section{APTITUD MUSICAL Y APTITUD LINGÜÍSTICA}

En la última década han surgido numerosos estudios desde distintas áreas científicas del conocimiento como la psicología, la neuropsicología, la educación general, la educación musical y la lingüística aplicada (enseñanza, aprendizaje y adquisición de lenguas) que describen las correlaciones entre música y lenguaje así como sus relaciones causales (Chobert \& Besson, 2013; Christiner \& Reiterer, 2013; Toscano-Fuentes \& Fonseca-Mora, 2012; Slevc \& Miyake, 2006).

Se evidencia que música y lenguaje comparten algunas destrezas auditivas como la discriminación melódica, rítmica y armónica y en general, la combinación y la segmentación de sonidos. Los procesos cognitivos que se emplean con la música para discriminar los sonidos y tonos, y asociar dichos sonidos con sus símbolos correspondientes, son similares a aquellos procesos de decodificación que, por ejemplo, se necesitan en el habla para reconocer y manejar los diferentes sonidos y descubrir qué composiciones forman palabras diferentes en el lenguaje hablado. Así, Fonseca-Mora, Toscano-Fuentes y Wermke (2011) definen la conexión entre música y lenguaje tanto en el proceso de adquisición de la lengua materna (L1) como en el proceso de adquisición de una segunda lengua (L2). Parten de la premisa que la adquisición de una lengua depende de la interacción y afirman que tanto el discurso de los padres como el de los maestros se asemejan en cuanto a las frecuentes repeticiones, el uso de expresiones lexicalizadas, las expansiones, la preferencia por un vocabulario simplificado, pero también por el cambio en el volumen de la voz así como las modificaciones de los contornos melódicos. Estas exageradas melodías en el habla han sido descritas como comportamientos intuitivos de los padres que guían a sus hijos en el aprendizaje musical (Papousek, 1996) pero que, a su vez, se establecen como elementos de comunicación que facilitan el aprendizaje de la L1 (Feu \& Piñero, 1996) y más en concreto, las melodías del llanto de los niños han sido definidas como soportes de la comunicación pre-verbal (Wermke \& Mende 2009). 
Cada idioma se caracteriza por elementos musicales muy específicos de su prosodia, es decir, su sistema de entonación y ritmo. La entonación, uno de los rasgos prosódicos más esenciales, proporciona la clave para la percepción de la acentuación de la palabra y el reconocimiento de la estructura de la oración. Esto es particularmente evidente en las primeras fases de adquisición de L1 donde la prosodia permite la segmentación del flujo del discurso audible y por lo tanto el reconocimiento de los elementos estructurales significativos. Las melodías son muy importantes ya para los fetos en el útero de sus madres como para los recién nacidos, ambos muestran una preferencia por las melodías con ritmos simples que caracterizan muchas piezas musicales infantiles así como la prosodia del discurso (Lecanuet et al., 1993).

En una etapa muy temprana del proceso de adquisición de la L1, los llantos, arrullos y balbuceos de los bebés se producen con una gran variedad de formas melódicas (Wermke \& Mende, 2006; 2009; Wermke, 2002). Wermke y Mende (2009), basándose en sus estudios longitudinales de los últimos 25 años, postulan que la melodía en el llanto de los bebés es un precursor del habla. Sus hallazgos se apoyan en la asunción que esas melodías funcionan como marcos y andamios durante la adquisición temprana de L1 y que evolucionan desde las melodías simples de las primeras semanas de vida a las primeras palabras habladas. Sus contribuciones se apoyan también en la observación de que el desarrollo perturbado de la melodía durante fases tempranas del discurso, antes de la adquisición de L1, se correlaciona con trastornos del lenguaje de los mismos niños 2,5 años más tarde (Wermke et al., 2007).

Estas ideas fundamentales con respecto a la función de andamiaje de las melodías durante fases tempranas de adquisición de L1 implican fuertes analogías para una eficaz adquisición de L2. Sleve y Miyake (2006) clasifican la habilidad musical individual como una de las diferencias individuales que afectan el nivel de adquisición de una lengua extranjera. Su estudio examinó la relación entre la capacidad musical y la competencia en una segunda lengua (L2) de estudiantes adultos. La competencia en L2 fue analizada desde el conocimiento léxico, la fonología productiva, la sintaxis y la fonología receptiva. También evaluaron otros factores que podrían explicar las diferencias individuales en la capacidad de L2, incluyendo la edad de inmersión de L2, los patrones de uso y exposición de la lengua, y la memoria fonológica a corto plazo. Según sus resultados, la habilidad musical predice la habilidad fonológica tanto receptiva y como productiva de la L2, es decir, afecta la comprensión oral y la pronunciación.

Según Ott et al. (2011), los músicos profesionales procesan los estímulos verbales o no de forma diferente dependiendo de su competencia musical. Esto se debe a las condiciones que afectan la plasticidad cerebral en las redes de procesamiento del habla, es decir al solapamiento anatómico en procesamiento de rasgos acústicos entre música y lenguaje, a la mayor precisión que exige el entrenamiento musical, a las respuestas emocionales, a la repetición y a la atención focalizada (Patel, 2011). Schön y Francois (2011) confirman con sus estudios electro-fisiológicos sobre la segmentación del habla y la extracción de información lingüística versus musical, que la competencia musical facilita tanto el aprendizaje de estructuras lingüísticas como musicales, mientras que Chobert y Besson (2013) aseguran que el efecto de la competencia musical influye en las habilidades lingüísticas de percepción y producción en una segunda lengua.

\section{¿EDUCACIÓN INSTRUMENTAL O ENSEÑANZA DE LENGUAS EXTRANJERAS CANTANDO?}

El rendimiento escolar tanto general como en una disciplina concreta no se debe a una única causa, sino que es más bien el resultado de la interacción de diversos factores como lo son, entre otros, la autoeficacia, las expectativas de logro, las habilidades objetivas, el componente genético, los rasgos de personalidad y las variables contextuales socioeconómicos, las culturales y las relacionadas con la institución educativa (Tenti, 2002). En el caso específico del aprendizaje de una lengua extranjera influye especialmente la motivación sostenida a lo largo del tiempo ya que se trata de un proceso de 
aprendizaje para el que los aprendices tildados en la literatura como "excepcionales” tardan más de dos años y medio o tres años. Entre todas estas variables mencionadas destaca la autoeficacia, la creencia que tiene una persona sobre su capacidad de poder lograr un determinado objetivo o no, lo que le hará persistir o no en el logro de su objetivo. Esta creencia que se tiene sobre nuestras posibilidades de realizar una determinada conducta con éxito determina en gran medida la actuación del individuo, resulta en ocasiones una variable que predice el rendimiento siendo incluso más fiable que el nivel real de la persona en esa habilidad concreta (Bandura, 1996; Pajares \& Kranzler, 1995; Multon, Brown \& Lent, 1991). Erler y Macaro (2011) analizan la relación entre las habilidades fonológicas necesarias para la lectura fluida de adolescentes estudiantes de francés como lengua extranjera y su autoeficacia. Su estudio muestra que la baja sensación de autoeficacia del alumnado incidía negativamente en su deseo de seguir estudiando esa lengua extranjera. Partiendo de las investigaciones que demuestran la relación entre autoeficacia y logro (Erler \& Macaro, 2011; Bandura, 2006), el presente estudio analiza la relación entre autoeficacia, formación musical y rendimiento académico en la asignatura de lengua extranjera inglés.

\section{AUTOEFICACIA, APTITUD MUSICAL E INGLÉS COMO LENGUA EXTRANJERA}

El rendimiento escolar tanto general como en una disciplina concreta no se debe a una única causa, sino que es más bien el resultado de la interacción de diversos factores como lo son, entre otros, la autoeficacia, las expectativas de logro, las habilidades objetivas, el componente genético, los rasgos de personalidad y las variables contextuales socioeconómicas, las culturales y las relacionadas con la institución educativa (Tenti, 2002). En el caso específico del aprendizaje de una lengua extranjera influye especialmente la motivación sostenida a lo largo del tiempo ya que se trata de un proceso de aprendizaje para el que los aprendices tildados en la literatura como "excepcionales” tardan más de dos años y medio o tres años. Entre todas estas variables mencionadas destaca la autoeficacia, la creencia que tiene una persona sobre su capacidad de poder lograr un determinado objetivo o no, lo que determinará el grado en el que persiste en el logro de su objetivo. Esta creencia sobre las propias posibilidades de realizar una determinada conducta con éxito determina en gran medida la actuación del individuo, resultando en ocasiones una variable predictora del rendimiento siendo incluso más fiable que el nivel real de la persona en esa habilidad concreta (Bandura, 1996; Multon, Brown y Lent, 1991; Pajares y Kranzler, 1995). Erler y Macaro (2011) analizan la relación entre las habilidades fonológicas necesarias para la lectura fluida de adolescentes estudiantes de francés como lengua extranjera y su autoeficacia. Su estudio muestra que la baja sensación de autoeficacia del alumnado incidía negativamente en su deseo de seguir estudiando esa lengua extranjera. Partiendo de las investigaciones que demuestran la relación entre autoeficacia y logro (Erler y Macaro, 2011; Bandura, 2006). En el presente estudio se analiza la relación entre autoeficacia, formación musical y rendimiento académico en la asignatura de lengua extranjera inglés.

\section{EL ESTUDIO: OBJETIVOS, PARTICIPANTES Y PROCEDIMIENTOS}

Ante la falta de conclusiones definitivas en contexto español sobre la relevancia de la formación musical instrumental y su beneficios para el aprendizaje de lenguas extranjeras, el objetivo central de este estudio fue establecer la relación entre aptitud musical y aprendizaje de inglés como LE en el alumnado español de ESO así como el de observar la percepción de autoeficacia en ILE de los dos grupos seleccionados. Se diseñó un estudio de correlación ex post facto que compara los resultados académicos de los alumnos de la ESO en el IES Lopez Neyra (Córdoba). Este IES desarrolla un programa piloto donde en una de sus líneas combina los estudios musicales y la enseñanza secundaria.

Participaron 454 estudiantes de ESO (primero a cuarto), 360 del régimen general de ESO y 94 del programa musical con el Conservatorio. Los alumnos del programa musical cursan las asignaturas optativas de la ESO en el Conservatorio Superior de Música en lugar de en el centro de enseñanza secundaria. El alumnado del programa musical ha de pasar el examen de selección para el ingreso en 
el conservatorio, por lo que se les presupone una aptitud musical elevada. Se analiza la correlación entre la condición "formación musical” dada por las calificaciones del "instrumento principal” y el rendimiento académico en la asignatura "inglés” en ESO. En una segunda fase del estudio se recoge información respecto al nivel de autoeficacia y el deseo de continuar estudiando inglés. La muestra de esta segunda es aleatoria y reducida (por razones de logística en la organización del centro).

\section{Preguntas de investigación}

Se plantearon tres preguntas de investigación para el estudio:

1. ¿Afecta la formación instrumental musical el aprendizaje de una lengua extranjera?

2. ¿Existe relación entre formación musical instrumental y sentido de la autoeficacia en una lengua extranjera?

3. ¿ ¿ncide la formación instrumental musical en la voluntad del alumnado de seguir estudiando la lengua extranjera?

\section{Objetivos}

Se establecieron los siguientes objetivos de investigación para dar respuesta a las preguntas:

1. Determinar la correlación entre las notas medias de los alumnos de ESO del centro y su vinculación bien al programa musical bien al programa general.

2. Determinar el nivel de autoeficacia de los alumnos y correlacionarlo con la condición: pertenencia al grupo "musical” o "general”.

3. Determinar el grado de voluntad del alumnado de continuar sus estudios de lengua extranjera y correlacionarlo con el nivel de autoestima y la pertenencia al grupo "musical” o "general"

\section{Método}

El estudio analiza la correlación entre la condición “formación musical” dada por las calificaciones del "instrumento principal”, variable independiente del estudio (VI) y el rendimiento académico en la asignatura "inglés”, variable dependiente (VD). En el mismo momento de recogida de información se recaban datos respecto al nivel de autoeficacia y el deseo de continuar estudiando inglés, variables que también se correlacionan con la VI. Se realiza un estudio de correlación entre las variables dependientes: "rendimiento escolar" y "autoeficacia”, donde se mantiene como variable independiente la aptitud musical. El mismo procedimiento se utiliza en la correlación final entre el deseo de seguir estudiando inglés (variable dependiente), nivel de autoeficacia y aptitud musical.

\section{Participantes}

Participaron 454 estudiantes de ESO (primero a cuarto), 360 del régimen general de ESO y 94 del programa musical con el Conservatorio. Los alumnos del programa musical cursan las asignaturas optativas de la ESO en el Conservatorio Superior de Música en lugar de en el centro de enseñanza secundaria. El alumnado del programa musical ha de pasar el examen de selección para el ingreso en el conservatorio, por lo que se les presupone una aptitud musical elevada, que funciona en el estudio como variable independiente (VI). 


\section{RESULTADOS}

El estudio comparativo de las medias de las notas finales obtenidas en inglés como lengua extranjera (ILE) por el alumnado a lo largo de los cuatro cursos de la ESO indica diferencias entre el alumnado participante en el programa musical del Conservatorio y el alumnado sin entrenamiento musical.

\begin{tabular}{|c|c|r|r|r|c|}
\hline & Música & $\mathrm{N}$ & Media & $\begin{array}{c}\text { Desvia- } \\
\text { ción } \\
\text { típica }\end{array}$ & $\begin{array}{c}\text { Error tí- } \\
\text { pico de la } \\
\text { media }\end{array}$ \\
\hline \multirow{2}{*}{ Notas } & no & 360 & 5,52 & 2,547 & 0,134 \\
\cline { 2 - 6 } & sí & 94 & 7,07 & 1,674 & 0,173 \\
\hline
\end{tabular}

Tabla 1: Rendimiento en la asignatura ILE de alumnado de ESO con y sin formación musical

Como se observa en la tabla 1, en un primer análisis, la media de los alumnos de la condición musical es superior a la de los alumnos en el programa general. No obstante, al ser poblaciones distintas se aplican la prueba de Levene para la igualdad de varianzas y la prueba $\mathrm{T}$ para la igualdad de medias (Tabla 2).

\begin{tabular}{|c|c|c|c|}
\hline \multicolumn{2}{|c|}{} & $\begin{array}{c}\text { Se han asumido varianzas } \\
\text { iguales }\end{array}$ & $\begin{array}{c}\text { No se han asumido } \\
\text { varianzas iguales }\end{array}$ \\
\cline { 2 - 4 } & F & Notas & Notas \\
\hline \multirow{2}{*}{$\begin{array}{c}\text { Prueba de Levene para la } \\
\text { igualdad de varianzas }\end{array}$} & Sig. & 24,163 & \\
\cline { 2 - 4 } & $\mathrm{t}$ & 0,000 & $-7,098$ \\
\cline { 2 - 4 } $\begin{array}{c}\text { Prueba T para la igualdad de } \\
\text { medias }\end{array}$ & gl & $-5,598$ & 218,824 \\
\cline { 2 - 4 } & Sig. (bilateral) & 452 & 0,000 \\
\cline { 2 - 4 } & Diferencia de medias & $-1,552$ & $-1,552$ \\
\hline
\end{tabular}

Tabla 2: Resultados de las pruebas de varianzas

Al indicarnos el estadístico aplicado (Prueba de Levene) que las varianzas son distintas (Sig < 0,05 ), se emplea la prueba $T$ para desigualdad de varianzas. Se encuentran diferencias significativas (Sig < 0,05) entre los que estudian música y entre los que no estudian música, con una diferencia de 1,552 puntos (sig < 0,05) a favor de los alumnos que a la condición "programa musical”.

El diseño inicial del estudio preveía el análisis de dos variables que en parte explicarán, caso de existir las diferencias encontradas. Se consideran primero las correlaciones entre autoeficacia, formación musical y rendimiento escolar en la asignatura de inglés para ver si existen diferencias significativas entre los alumnos con formación musical y los que no la tienen, los resultados se muestran en la tabla 3. 


\begin{tabular}{|c|c|c|c|}
\hline \multirow{2}{*}{} & $\begin{array}{c}\text { Alumnos sin } \\
\text { formación musical. } \\
\text { Correlaciones }\end{array}$ & $\begin{array}{c}\text { Alumnos con } \\
\text { formación musical. } \\
\text { Correlaciones }\end{array}$ \\
\cline { 3 - 4 } & \multicolumn{2}{|c|}{ Autoeficacia } \\
\hline \multirow{3}{*}{ Nota_Inglés } & $\begin{array}{c}\text { Correlación de } \\
\text { Pearson }\end{array}$ & 0,358 & 0,505 \\
\cline { 2 - 4 } & Sig. (bilateral) & 0,072 & 0,000 \\
\cline { 2 - 4 } & $\mathrm{N}$ & 26 & 47 \\
\hline
\end{tabular}

Tabla 3: Resultados de correlaciones formación musical, autoeficacia y rendimiento escolar en ILE

Las correlaciones sólo muestran una relación significativa entre la autoeficacia y el rendimiento escolar en inglés del alumnado que recibe formación musical, se observa el coeficiente $\rho<0,5 \mathrm{y}$ p $>0,05$ en los alumnos sin formación musical, mientras que en los alumnos conformación musical el coeficiente es $>0,5$ y $\mathrm{p}<0,05$.

El análisis concreto de la pregunta final del cuestionario, que los interpelaba sobre su deseo de seguir estudiando inglés, permite observar su correlación con la formación musical. Se realiza en esta ocasión un test no paramétrico al ser la variable "pregunta número 13”, categórica de tipo ordinal.

\begin{tabular}{|c|c|c|c|c|}
\hline \multicolumn{2}{|l|}{} & $\begin{array}{c}\text { Alumnos sin } \\
\text { formación } \\
\text { musical. }\end{array}$ & $\begin{array}{c}\text { Alumnos con } \\
\text { formación } \\
\text { musical. }\end{array}$ \\
\hline Rho de Spearman & Nota_Inglés & $\begin{array}{c}\text { Coeficiente de } \\
\text { correlación }\end{array}$ & 0,173 & 0,47 \\
\hline & & Sig. (bilateral) & 0,398 & 0,001 \\
\hline & & $\mathrm{N}$ & 26 & 47 \\
\hline
\end{tabular}

Tabla 4: Intención de seguir estudiando ILE

Como se observa en la tabla 4, no existe relación en el caso de los alumnos sin formación musical el coeficiente $(\rho<0,5$ y p $>0,05)$ mientras que para el alumnado con formación musical se relaciona significativa su percepción de autoeficacia en el aprendizaje de la lengua extranjera, inglés, y su deseo de continuar estudiándola en el futuro $(\rho>0,5$ y $p<0,05)$.

\section{DISCUSIÓN Y CONCLUSIÓN}

Actualmente existe un creciente auge de investigaciones internacionales que explican los beneficios de la formación musical en el currículum académico. No constituye solo la posibilidad de un hobby para el ocio, lo cual ya por sí es muy relevante, sino que además afecta nuestras redes neuronales y presenta beneficios en los procesos cognitivos.

Según los resultados obtenidos en este estudio, la formación en competencias musicales instrumentales incide positivamente en el aprendizaje de inglés como LE de alumnado español de ESO. El alumnado de ESO con formación musical instrumental obtiene mejores resultados académicos en inglés. Esto está en consonancia con los solapamientos neuronales entre música y lenguaje que explica Patel (2011). Tal como afirman Sleve y Miyake (2006), la competencia musical Individual constituye una de las diferencias individuales que afectan el nivel de adquisición de una lengua extranjera. Su estudio examinó la relación entre la capacidad musical y la competencia en una segunda lengua (L2) de estudiantes adultos, mientras que este estudio contribuye con la importancia de la formación instrumental musical de alumnado entre 12 y 16 años de edad. En nuestro estudio, la creencia de los adolescentes sobre su capacidad de aprender una lengua extranjera es superior a la del resto del alumnado de ESO sin formación musical, lo que además incide en su voluntad de seguir estudiando esta lengua extranjera. Esto supone un dato relevante a la hora de diseñar la organización de las materias que han de abordarse en la escolaridad obligatoria si se busca una sociedad con posibilidades igualitarias de desarrollo, la competencia comunicativa en inglés como lingua franca 
es una de las competencias básicas actuales del siglo XXI. La inclusión del entrenamiento musical instrumental según nuestro estudio, o vocal según confirman los estudios de Christener y Reiterer (2013), no solo ayuda al alumnado a mejorar su pronunciación en una lengua extranjera sino que afecta a todo su sistema de creencias sobre la capacidad de lograrlo o no y por lo tanto, a la motivación sostenida que es imprescindible en el aprendizaje de lenguas.

\section{Referencias}

Alipour, M., Gorjian, B., \& Zafari, I. (2012). The effects of songs on EFL learners' vocabulary recall and retention: The case of gender. Advances in Digital Multimedia, 1(3), 140-143.

Bandura, A. (1997). Self-efficacy: The exercise of control. Nueva York: W. H. Freeman.

Butzlaff, R. (2000). Can music be used to teach reading? Journal of Aesthetic Education, 34, 3-4: $167-78$.

Chobert, J., \& Besson, M. (2013). Musical expertise and second language learning. Brain Sciences, 3(2), 923-940. doi:10.3390/brainsci3020923

Christiner, M., \& Reiterer, S. M. (2013). Song and speech: examining the link between singing talent and speech imitation ability. Frontiers in Psychology, 4, 874 doi: 10.3389/fpsyg.2013. 00874.

Feu Guijarro, M. J. \& Piñero Gil, E. (1996). El mundo sonoro infantil y la adquisición del lenguaje. Música, Arte y Proceso, 2, 39-49.

Fonseca-Mora, M. C., Toscano-Fuentes, C., \& Wermke, K. (2011). Melodies that help: The relation between language aptitude and musical intelligence. Anglistik: International Journal of English Studies 22(1), 101-118

Kang, H. J. \& Williamson, V. J. (2014). Background music can aid second language learning. Psychology of Music, 42, 5: 728-747 doi:10.1177/0305735613485152

Lecanuet, J. P., Granier-Deferre, C., Jacquet, A. I., Capponi, I., \& Ledru, L. (1993). Prenatal discrimination of a male and a female voice uttering the same sentence. Early Development and Parenting, 2, 217-228.

Lessard, A., \& Bolduc, J. (2011). Links between musical learning and reading for first to third grade students: A literature review. International Journal of Humanities and Social Science, 1, 7.

Ludke, K. M., Ferreira,F., \& Overy, K. (2014). Singing can facilitate foreign language learning. Memory \& Cognition, 42(1), 41-52. doi:10.3758/s13421-013-0342-5

Moritz, C., Yampolsky, S., Papadelis, G., Thomson, J., \& Wolf, M. (2012). Links between early rhythm skills, musical training, and phonological awareness, Reading and Writing, 26(5), 131. doi:10.1007/s11145-012-9389-0

Multon, K. D., Brown, S. D., \& Lent, R. W (1991). Relation of self efficacy beliefs to academic outcomes: A meta analytic investigation. Journal of Counseling Psychology, 38, 30-38.

Ott, C. G. M., Langer, N., Oechslin, M. S., Meyer, M., \& Jäncke, L. (2011). Processing of voiced and unvoiced acoustic stimuli in musicians. Frontiers in Psychology, 2, 195 doi: 10.3389/fpsyg.2011.00195

Pajares, F., \& Kranzler, J. (1995). Competence and confidence in mathematics: The role of selfefficacy, self-concept, anxiety, and ability. Comunicación presentada en la Annual Meeting of the American Educational Research Association. San Francisco, California. 
Papousek, M. (1996). Intuitive parenting. En I. Deliège \& J. Sloboda (Eds.). Musical beginnings: Origins and development of musical competence (págs. 88-108). Oxford: Oxford University Press, 88-108.

Patel, A. D. (2011). Why does musical training benefit the neural encoding of speech? A new hypothesis. The Journal of the Acoustical Society of America, 130(4), 2398 dx.doi.org/10.1121/1.3654612

Ros, M. T. S. (2005). La reducción de la ansiedad en el aprendizaje de una lengua extranjera en la enseñanza superior: el uso de canciones. Interlingüística, 16, 1029-1039.

Schön, D., \& François, C. (2011). Musical expertise and statistical learning of musical and linguistic structures. Frontiers in Psychology, 2, 167. doi: 10.3389/fpsyg.2011.00167

Slevc, L. R., \& Miyake, A. (2006). Individual differences in second language proficiency: Does musical ability matter? Psychological Science, 17(8), 675-681. doi: 10.1111/j.14679280.2006. 01765.x

Standley, Jayne M. (2008). Does music instruction help children learn to read? Evidence of a metaanalysis. Update: Applications of Research in Music Education, 27(1), 17-32. doi: $10.1177 / 8755123308322270$

Tenti, E. (2002). El rendimiento escolar en la Argentina. Buenos Aires: Losada.

Toscano-Fuentes, C. M., y Fonseca-Mora, M. C.(2012). La música como herramienta facilitadora del aprendizaje del inglés como lengua extranjera. Teoría de la Educación, 24(2), 197-213.

Wermke, K. (2002). Untersuchung der Melodieentwicklung im Säuglingsschrei von monozygoten Zwillingen in den ersten 5 Lebensmonaten. Tesis de Habilitación, Humboldt-Universität zu Berlin, http://edoc.hu-berlin.de

Wermke, K. \& Mende, W. (2006). Melody as a primordial legacy from early roots of language. Behavioral and Brain Sciences, 29, 300. doi: 10.1017/S0140525X06449062

Wermke, K., Leising, D., \& Stellzig-Eisenhauer, A. (2007). Relation of melody complexity in infants' cries to language outcome in the second year of life: A longitudinal study. Clinical Linguistics and Phonetics, 21, 961-973. doi:10.1080/02699200701659243

Wermke, K., \& Mende, W. (2009). Musical elements in human infants' cries: In the beginning is the melody. Musicae Scientiae, 13(2 suppl.), 151-175. doi: 10.1177/1029864909013002081 